\title{
Gut Microbiota Dysbiosis Drives the Development of Colorectal Cancer
}

\author{
Xiaoyan Fan ${ }^{\mathrm{a}, \mathrm{b}}$ Yuelei Jin ${ }^{\mathrm{a}}$ Guang Chen ${ }^{\mathrm{a}} \quad$ Xueqiang Ma ${ }^{\mathrm{c}}$ Lixia Zhang ${ }^{\mathrm{b}}$ \\ aDepartment of Basic Medical Sciences, Taizhou University Hospital, Taizhou University, Taizhou, China; \\ ${ }^{b}$ Department of Neurology, Taizhou Second People's Hospital, Taizhou, China; 'Department of Gastrointestinal \\ Surgery, Municipal Hospital Affiliated to Medical School of Taizhou University, Taizhou, China
}

\section{Keywords}

Gut microbiota dysbiosis · Pathogenic bacteria · Colorectal cancer - Tumorigenesis - Diagnostic biomarker

\begin{abstract}
Background: The gut microbiota is a diverse community of microbes that maintain the stability of the intestinal environment. Dysbiosis of the gut microbiota has been linked to gastrointestinal diseases, such as colorectal cancer (CRC) - a leading cause of death for cancer patients. Summary: Candidate pathogens have been identified using bacterial culture and high-throughput sequencing techniques. Currently, there is evidence to show that specific intestinal microbes drive CRC development and progression, yet their pathogenic mechanisms are still unclear. Key Messages: In this review, we describe the known healthy gut microbiota and its changes in CRC. We especially focus on exploring the pathogenic mechanisms of gut microbiota dysbiosis in CRC. This is crucial for explaining how gut microbiota dysbiosis drives the process of colorectal carcinogenesis and tumor progression. Evaluation of changes in the gut microbiota during CRC development and progression offers a new strategy for the diagnosis and treatment of this disease.
\end{abstract}

\section{Introduction}

The human gut microbiota is the collection of various microbes that inhabit the normal intestinal tract. The total number of microbial cells throughout the intestinal tract reaches $10^{13}$ to $10^{14}[1]$, including bacteria, viruses, fungi, and archaea, among which bacteria are predominant. To date, approximately 1,000-1,150 bacterial species have been identified in the human intestine, and the core microbiota shared by different individuals comprises $~ 160$ species [2]. The gut microbiota is mainly composed of 4 phyla: Firmicutes, Bacteroidetes, Actinobacteria, and Proteobacteria [3]. Various microbial species cooccur in specific proportions, interdependent on and competitive to each other, which maintain a relatively stable ecology [1].

The gut microbiota can be classified into 3 clusters according to their influence on the human body: beneficial, neutral, and pathogenic. Beneficial microbes, such as $B i$ fidobacterium and Lactobacillus, can protect the intestinal tract, produce beneficial metabolites, and detoxify the human gut [4]. Neutral microbes, such as Enterococcus,

X.F. and L.Z. contributed equally to this work.

Yuelei Jin

Department of Basic Medical Sciences

Taizhou University, No. 1139 Shifu Road

Jiaojiang District, Taizhou, Zhejiang Province 318000 (China)

jinyuelei_001@163.com 
have dual characters; they are beneficial to human health in normal growth conditions and may cause different degrees of diseases when exceeding a certain growth standard or transferred to other parts of the body [5]. Pathogenic microbes, such as Salmonella and Helicobacter pylori, secrete toxins in the intestinal tract and thus harm human health [6]. Moreover, the gut microbiota can be divided into 3 groups based on their oxygen demand, obligately anaerobic, facultatively anaerobic, and aerobic, among which anaerobic bacteria are most dominant [7].

In normal conditions, the gut microbiota plays a pivotal role in bodily functions, such as promoting the digestion and absorption of nutrients, maintaining the normal physiological function of the intestinal tract, and modulating the immunity. When the gut microbiota is influenced by multifactors including the diet, environment, and host genes, its dysbiosis is manifested as altered microbiota composition, bacterial bioactivity, and location in different parts of the human body [8]. Such alterations may cause diseases, such as inflammatory bowel disease, colorectal cancer (CRC), cardiovascular disease, diabetes mellitus, and mental illness $[9,10]$. However, the causal relationship between the gut microbiota and these diseases has not yet been clarified.

CRC is the third most common malignancy that seriously threatens human health. It has become a leading cause of death among cancer patients worldwide [11] and is responsible for nearly 500,000 deaths per year, posing a major global challenge for healthcare. In China, 376,000 new cases and 191,000 deaths occurred annually from 2009 to 2011 on average [12], making CRC the fifth leading cause of cancer-related deaths. This incidence could be even higher in economically developed cities [13]. $\mathrm{CRC}$ is a malignant disease involving multifactors. A large body of work has indicated that the etiology of CRC includes genetic background and environmental risk factors, such as diabetes mellitus, cholecystectomy, obesity, high-fat diet, processed food, and red meat [14-17]. In the past decade, researchers have provided massive important information on the role of intestinal microbes in CRC development and progression. The causal relationship between the presence of specific microbes and CRC development has also been verified.

\section{Discussion}

Specific Microbes Associated with CRC

With the emergence of numerous new techniques, large-scale genetic and metabolic analyses of the gut mi- crobiota have been conducted, which provide insight into the role of human gut microbiota. The relationship between gut microbiota dysbiosis and CRC has been implicated in a growing number of studies [18-23]. The primary characteristics of microbiota dysbiosis are alteration of bacterial species and increase of pathogenic bacteria. Evidence suggests that microbiota dysbiosis can cause alteration of the host's physiological functions and thereby lead to the pathogenesis of various diseases $[24,25]$. Significant dysbiosis of microbes often occurs in CRC tissues and adjacent mucosa [26]. However, due to the different classification standards and methods used in past studies, the key species involved in CRC progression are still unclear. This raises difficulties in summing up the specific characteristics of CRC-related intestinal microbes. Here we provide a summary of CRC-related gut bacteria that have been reported frequently as of date.

\section{Fusobacterium nucleatum}

Fusobacterium nucleatum (Fn) is a Gram-negative, anaerobic bacterium [27]. As a member of the oral microbiota, $F n$ is commonly found in periodontal disease and rarely detected in the normal human intestinal tract [28]. Based on quantitative PCR, 16S rRNA sequencing, and FISH analyses, the level of $F n$ was found to increase in adenomas and CRC compared with healthy controls, and high $F n$ level was associated with lymph node metastasis of CRC [18]. Chen et al. [19] found that $F n$ can drive CRC progression due to its ability to inhibit the antitumor immune response through reducing the number of $\mathrm{CD} 4^{+} \mathrm{T}$ cells and downregulating the expression of the TOX protein. However, Fn alone cannot colonize the colon, as it requires interaction with several other species to form colonies. Fn secretes the Fad A adhesin that binds to the extracellular region of E-cadherin in epithelial cells to stimulate the catenin pathway, leading to the activation of inflammatory response and carcinogenesis [29]. Additionally, E-cadherin increases the expression of transcription factors, Wnt genes, and inflammatory genes by upregulating the Wnt pathway, which in turn stimulates the growth of CRC cells [30]. Furthermore, Fn secrets the Fap 2 protein that targets the inhibitory receptor TIGIT (T-cell immunoglobulin and immunoreceptor tyrosinebased inhibitory motif domain), which then acts on $\mathrm{T}$ cells and natural killer cells; this action inhibits T cell activation and natural killer cell-mediated tumor cell killing, facilitates tumor cell proliferation, and thus results in CRC development [31]. 
Enterotoxigenic Bacteroides fragilis

Enterotoxigenic Bacteroides fragilis $(E T B F)$ is a Gramnegative, anaerobic bacterium [20]. It produces $B$. fragilis toxin (BFT) to participate in inflammation-related diarrhea or inflammation-induced tumor formation [20]. BFT is a zinc-dependent metalloproteinase toxin that binds to receptors on colonic epithelial cells, thereby increasing mucosal permeability and cytokine secretion; it then participates in various signal transduction in colonic epithelial cells, such as upregulating the Wnt pathway and proinflammatory MAPK signals, driving the structure of intestinal epithelial mucosal tissue to develop via the inflammation-adenoma-adenocarcinoma pathway [32]. A mouse experiment conducted by Boleij et al. [33] showed that BFT can aggravate intestinal inflammatory response and tumor progression; it was also demonstrated that BFT and Th17/IL-17 play an essential role in the process of $E T B F$-induced carcinogenesis. Moreover, BFT enhances the differentiation of bone marrow cells into myeloid-derived suppressor cells and then induces CRC development through pathogenic inflammatory pathways [34].

\section{Escherichia coli}

E. coli is a human gut commensal that belongs to Gram-negative, anaerobic bacteria [35]. Swidsinski et al. [36] showed that E. coli is able to colonize the colonic mucosa; it increases mucosal permeability through activation of Wnt mitogenic signaling, DNA damage and interference with the DNA repair process, and dysfunction of E-cadherins, thereby inducing CRC development [7, 37, 38]. E. coli can be divided into at least 5 subtypes: A, B1, $\mathrm{B} 2, \mathrm{D}$, and $\mathrm{E}$ [39]. According to a study involving xenografts and inflammation-related animal tumor models [21], the subtype B2 of E. coli harbors a conserved gene island - "polyketide synthase ( $p k s)$," which produces peptide-polyketide colibactin. This genotoxin can penetrate colonic cell membranes and migrate to the nucleus, where it causes DNA double-strand breaks, cell cycle arrest, and incomplete DNA repair, giving rise to chromosomal aberrations and then carcinogenesis [9-11]. pks can induce carcinogenesis by damaging DNA [40-42]. In another mouse experiment, knocking out the pks gene island mitigated DNA damage and reduced the amount of bacterial infection and tumor formation, without any inflammatory response; this further confirms that the pks gene island can induce CRC development [22]. The role of E. coli in CRC was also supported by a pathologic analysis in a French population of 50 patients with adenocarcinoma and 33 with diverticulosis [43].

\section{Salmonella}

Salmonella is a group of Gram-negative, facultatively anaerobic bacteria, which are common pathogens found in humans and animals [44]. Common sources of Salmonella infection include contaminated foods, such as meat, eggs, and agricultural products [45]. Following Salmonella infection, some chronic complications may manifest, including reactive arthritis, irritable bowel syndrome, inflammatory bowel disease, or carcinogenesis [46, 47]. A study conducted in American and Dutch populations by Kato et al. [23] showed that the expression levels of Salmonella flagella antibodies were significantly higher in CRC patients and precancerous lesions than in healthy controls, with dietary intake being one of the mediating factors; this is suggestive of a potential link between Salmonella and CRC [23]. Salmonella can secrete the effector protein AvrA to promote acetylation and ubiquitination of target proteins. AvrA inhibits $\beta$-catenin degradation, maintains $\beta$-catenin stability, and promotes intestinal epithelial cell proliferation, thereby facilitating tumorigenesis. Moreover, AvrA increases tumor diversity and drives tumor progression $[48,49]$.

\section{Other Bacteria}

Desulfovibrio desulfuricans[50] and Enterococcus faecalis [51] are Gram-negative and Gram-positive anaerobic bacteria, respectively. These bacteria may be associated with CRC because their metabolites (hydrogen sulfide and superoxide radical anions) are potentially detrimental to colorectal mucosa [52]. Moreover, Khan et al. [53] reported a case of anaerobic bacteremia with increased level of Parvimonas micra, which was found to be local right-sided colon cancer by early examination. Yu et al. [54] observed an enrichment of Solobacterium moorei and CRC-related genes in the early stage of CRC. Together, these studies suggest that $P$. micra and $S$. moorei are likely to be associated with CRC development.

\section{Microbial Metabolites Associated with CRC}

Short-Chain Fatty Acids

Gut bacteria (e.g., Clostridium cluster IV and Fecalibacterium) produce short-chain fatty acids (SCFAs) through fermentation of dietary fiber, and the products, including butyrate, acetate, propionate, and valerate, act as regulators of immune response [55]. SCFAs can support the health of gut microbiota and increase the microbial diversity. Additionally, SCFAs play a positive role in the intestinal barrier function by promoting mucus production and connexin expression. Furthermore, SCFAs can reduce the oxygen concentration in the intestinal cav- 
Table 1. Mechanisms of action of differential intestinal bacteria in the development and progression of colorectal cancer

\begin{tabular}{llll}
\hline Mechanism & Bacterial species & References \\
\hline Inflammatory response & $\begin{array}{l}\text { Alistipes finegoldii, Bacteroides fragilis, Bacteroides vulgatus, Padenia } \\
\text { intermedia, Enterococcus faecalis, Eubacterium ventriosum, } \\
\text { Parvimonas micra, Streptococcus gallolyticus, Fusobacterium } \\
\text { nucleatum, Helicobacter pylori }\end{array}$ & {$[68-76]$} \\
\hline Immune response & F. nucleatum, P. micra, Akkermansia muciniphila & {$[31,77-81]$} \\
\hline DNA damage & $\begin{array}{l}\text { Escherichia coli, enterotoxigenic Bacteroides fragilis, Enterococcus spp., } \\
\text { Escherichia spp. }\end{array}[21,22,41,72,82-85]$ \\
\hline Modulation of cell proliferation & C. rodentium, enterotoxigenic B. fragilis, F. nucleatum & {$[30,86,87]$}
\end{tabular}

ity and help to maintain a healthy immune system. Due to these functions, SCFAs have a preventive effect on CDC development [56]. It has been found that SCFAs inhibit colitis and associated carcinogenesis by binding to the receptor Gpr109a [57]. Butyrate, as one of the most important members of the SCFA family, has anti-inflammatory and antitumor properties through cell metabolism, microbiota homeostasis, immune regulation, and gene epigenetic modulation [56]. A study has shown that butyric acid drives apoptosis in CDC cells by inhibiting the expression of histone deacetylase-regulating genes [58].

\section{Other Metabolites}

Other metabolites of gut bacteria, including secondary bile acids, deoxycholic acid, and lithocholic acid, have also been shown to be related to CRC development [59]. Anaerobic gut bacteria decompose the bile acids remaining in the intestine into deoxycholic acid and lithocholic acid, both of which can cause DNA damage by generating free radicals and thus increase the risk of CRC development [60]. Primary and secondary bile acids are reportedly associated with CDC [61-63]. A high-fat diet can lead to an increase in bile acid synthesis in the liver, which in turn increases bile acid accumulation in the intestine. Primary bile acids are converted into secondary bile acids through 7- $\alpha$ dehydroxylase produced by gut microbiota. Secondary bile acids, especially deoxycholic acid, are considered to be genotoxic since they can cause DNA damage in intestinal mucosal epithelial cells [64] and facilitate the development and progression of intestinal tumors [65]. Moreover, gut bacteria produce several harmful compounds that may play a role in CDC and inflammatory bowel disease. For example, ammonia, phenols, $p$-cresol, certain amines, and hydrogen sulfide are likely to partici- pate in the onset or deterioration of cancers through chronic inflammation and DNA damage [66].

Mechanisms of Gut Microbiota Dysbiosis in Driving CRC Development

In recent years, with the advance of culture technique, high-throughput sequencing, and multiomics research, evidence is amounting that gut microbiota dysbiosis is closely associated with CRC development and progression $[7,67]$. The possible mechanisms by which gut microbiota dysbiosis drives CRC development are summarized in Table 1.

\section{Inflammatory Response}

In the normal gut, the interaction between commensal bacteria and immune cells constitutes a delicate balance: proinflammatory genes and oncogenes versus anti-inflammatory genes and antioncogenes [88]. In the case of gut microbiota dysbiosis, it has been found that inflammatory microenvironment-mediated activation of inflammatory pathways drives CRC development and progression. Immune cells in the microenvironment and the cytokines and growth factors they produce activate associated signaling pathways, such as Wnt, Notch, and TGF- $\beta$, which in turn affect the self-renewal of colonic mucosal epithelial cells. These components also activate the transcription factors NF-KB and STAT3, thus influencing colonic tissue repair and immune homeostasis. Moreover, they activate the MAPK and Akt/PKB pathways, which affects mitosis and survival of colonic cells [89]. ETBF can elevate chemokine L20 and prostaglandin E2 levels in intestinal epidermal cells; prostaglandin E2 participates in the proliferation and activation of Th17 cells and enhances the secretion of IL-17 and related factors by Th17 cells, leading to the development of inflam- 
mation-related CRC $[90,91]$. Microbial products are sensed by Toll-like receptors, which trigger MyD 88-mediated production of proinflammatory cytokine IL-23. IL-23 activates IL-17a, IL-6, and IL-22 production and thereby promotes CRC development [92]. Moreover, gut microbiota dysbiosis induces the production of tumorrelated genotoxins and metabolites, which in turn causes immune response disorders, promotes and maintains inflammatory bowel disease, and leads to CRC development.

\section{Immune Response}

The close contact between the gut microbiota and the mucosal immune system also stimulates tumorigenic immune responses through endogenous bacteria. Th17 immunity is usually carcinogenic and is associated with a poor prognosis of CRC; its activation is driven by microbes and their products [92]. For example, Fn uses Fap2 for cell adhesion and it directly interacts with $\mathrm{T}$ cell immunosuppressive receptors through TIGIT, which results in the loss of function of tumor-killing lymphocytes [31]. Second, Fn modulates the enrichment of myeloidderived suppressor cells and tumor-associated macrophages, both of which inhibit antitumor $\mathrm{T}$ cell response [81].

\section{DNA Damage}

Accumulation of DNA damage can cause destruction of genome stability in the host and then promote precancerous and cancerous cells to maintain or accelerate mutations [93]. Intestinal microbes are exactly a potential source of DNA mutagens. In vitro and in vivo studies showed that some Enterococcus spp. produce reactive oxygen species, such as hydroxyl free radicals [83]; these substances can cause DNA breaks, point mutations, and protein-DNA crosslinking, reducing the stability of CRCrelated genes [84]. Various animal models of CRC also demonstrated that ETBF induces the expression of spermine oxidase on the colonic epithelium; this enzyme can induce DNA damage [40]. Additionally, pks-positive E. coli promotes DNA double-strand breaks, cell cycle arrest, and aneuploid cell division in vivo [21, 22, 82]. Recently, it was also found that the differential abundance of several gut bacteria is associated with NRAS, TP53, and RAS mutations in the host, based on $16 \mathrm{~S}$ rRNA gene sequencing of fecal bacteria in $83 \mathrm{CRC}$ patients and 60 normal controls from Iran and Finland [94]. Microbes directly or indirectly cause DNA damage and influence genome stability, making the microbiome a potential risk factor and therapeutic target for CRC.
Modulation of Cell Proliferation

The growth and death-inducing signals are modulated strictly in normal tissues to maintain the cell structure and function. Once disturbances of cell proliferation and apoptotic signals cause continued cell proliferation, eventually malignant tumors will form [95]. Citrobacter rodentium-infected mice can mediate Wnt- $\beta$-catenin activation through $\mathrm{R}$-spondin 2 , resulting in the overproliferation and poor differentiation of intestinal stem cells [86]. ETBF releases BFT to promote E-cadherin cleavage, and it also facilitates nuclear translocation of $\beta$-catenin, transcriptional upregulation of the proto-oncogene $c$ $M y c$, and colonic epithelial proliferation [87]. Through a similar mechanism, Fn binds to E-cadherin through Fad A, then activating downstream pathways and facilitating epithelial cell proliferation [30]. In summary, past studies indicated that distorted gut microbiota is a signal source that activates abnormal epithelial cell proliferation and drives early tumor progression.

\section{Specific Microbiota as a Diagnostic Biomarker for CRC}

Patients with CRC and healthy individuals possess significantly different gut microbiota in their feces [96]. Hence, gut microbiota may serve as a microbial marker for the risk of developing CRC, which can be used to screen CRC and precancerous lesions. In an earlier study, Zackylar et al. [97] used high-throughput sequencing to screen differential bacterial genera in fecal specimens collected from 30 healthy controls, 30 patients with colon adenoma, and 30 patients with CRC in England and then built a microbial prediction model. The proposed model coupled with CRC-related risk factors substantially improved the ability to distinguish between healthy controls, adenomas, and CRC, achieving a 50-fold increase in the detection rate of CRC. Subsequently, Lee et al. [98] and Komiya et al. [99] tested 40 genes and 5 signal pathways in 2 groups of CRC patients ( $F n$-high and $F n$-low). They observed that the mutation rates of AMER1 and ATM genes and TGF- $\beta$ pathway were higher in the Fnhigh group than in the Fn-low group, and Fn-high was related to poor prognosis of CRC. More recently, Colov et al. [100] found that high levels of $F n$ and possibly $B$. fragilis were associated with worse prognosis after surgery for CDC. Further, a multivariate analysis confirmed that $F n$-high was an independent negative prognostic factor for CRC.

Yu et al. [54] found that apart from Fn and Peptostreptococcus stomatis that were identified previously, P. micra, S. moorei, and CRC-related genes were enriched in 
the early stage of CRC. These species, therefore, can be used as an early diagnostic tool for CRC to accurately distinguish CRC patients from healthy population. Shah et al. [101] analyzed raw 16S rRNA gene sequence data of the fecal microbial community from 9 CRC studies. They found that specific microbiota including P. micra ATCC 33270 and Streptococcus were increased significantly in CRC patients, which could effectively distinguish between CRC patients and healthy individuals. Despite technical differences in various study methods or bioinformatics processes, the key microbial markers are crucial for the classification of CRC cases and they can be used for the general diagnosis of CRC disease [101].

\section{Conclusion}

The gut microbiota systematically participates in the nutrition, metabolism, and immune functions of the human body. Gut microbiota dysbiosis causes an imbalance in microbial homeostasis, while specific microbiota induces CRC development and facilitates its progression via different pathways, such as driving inflammatory response, inducing DNA damage, and/or promoting cell proliferation. Species differences in the gut microbiota during tumorigenesis can be used as a biomarker and diagnostic tool for CRC. It is expected to find specific microbial species and their metabolites closely related to CRC development and progression by further research, which will provide new strategies for the prediction, prevention, and clinical research of CRC.

\section{Conflict of Interest Statement}

The authors have no conflicts of interest to declare.

\section{Funding Sources}

There are no funding sources to declare.

\section{Author Contributions}

X.F. and L.J. conceived and designed the study, drafted the article, and approved the final version. G.C. and X.M. designed the study, critically revised the article, and approved the final version. L.Z. conceived and designed the study, critically revised the article, and approved the final version.

\section{References}

1 Gill SR, Pop M, Deboy RT, Eckburg PB, Turnbaugh PJ, Samuel BS, et al. Metagenomic analysis of the human distal gut microbiome. Science. 2006;312(5778):1355-9.

2 Qin J, Li R, Raes J, Arumugam M, Burgdorf KS, Manichanh C, et al. A human gut microbial gene catalogue established by metagenomic sequencing. Nature. 2010;464(7285): $59-65$.

3 Khanna S, Tosh PK. A clinician's primer on the role of the microbiome in human health and disease. Mayo Clin Proc. 2014;89(1):107-14.

4 Kich DM, Vincenzi A, Majolo F, Volken de Souza CF, Goettert MI. Probiotic: effectiveness nutrition in cancer treatment and prevention. Nutr Hosp. 2016;33(6):1430-7.

5 The Human Microbiome Project Consortium. A framework for human microbiome research. Nature. 2012;486(7402):215-21.

6 Peterson DA, Frank DN, Pace NR, Gordon JI. Metagenomic approaches for defining the pathogenesis of inflammatory bowel diseases. Cell Host Microbe. 2008;3(6):417-27.

7 Gagnière J, Raisch J, Veziant J, Barnich N, Bonnet R, Buc E, et al. Gut microbiota imbalance and colorectal cancer. World J Gastroenterol. 2016;22(2):501-18.

8 Sun J, Kato I. Gut microbiota, inflammation and colorectal cancer. Genes Dis. 2016;3(2): 130-43.
9 Jackson MA, Verdi S, Maxan ME, Shin CM, Zierer J, Bowyer RCE, et al. Gut microbiota associations with common diseases and prescription medications in a population-based cohort. Nat Commun. 2018;9(1):2655.

10 Hollister EB, Gao C, Versalovic J. Compositional and functional features of the gastrointestinal microbiome and their effects on human health. Gastroenterology. 2014;146(6): 1449-58.

11 Miller KD, Siegel RL, Lin CC, Mariotto AB, Kramer JL, Rowland JH, et al. Cancer treatment and survivorship statistics, 2016. CA Cancer J Clin. 2016;66(4):271-89.

12 Chen W, Zheng R, Baade PD, Zhang S, Zeng $\mathrm{H}$, Bray F, et al. Cancer statistics in China, 2015. CA Cancer J Clin. 2016;66(2):115-32.

13 Chen W, Zheng R, Zeng H, Zhang S, He J. Annual report on status of cancer in China, 2011. Chin J Cancer Res. 2015;27(1):2-12.

14 Larsson SC, Orsini N, Wolk A. Diabetes mellitus and risk of colorectal cancer: a meta-analysis. J Natl Cancer Inst. 2005;97(22):1679-87.

15 Larsson SC, Wolk A. Meat consumption and risk of colorectal cancer: a meta-analysis of prospective studies. Int J Cancer. 2006; 119(11):2657-64.

16 Shao T, Yang YX. Cholecystectomy and the risk of colorectal cancer. Am J Gastroenterol. 2005;100(8):1813-20.
17 Gonder U, Worm N. Re: meat, fish, and colorectal cancer risk: the European prospective investigation into cancer and nutrition. J Natl Cancer Inst. 2005;97(23):1788-9.

18 Castellarin M, Warren RL, Freeman JD, Dreolini L, Krzywinski M, Strauss J, et al. Fusobacterium nucleatum infection is prevalent in human colorectal carcinoma. Genome Res. 2012;22(2):299-306.

19 Chen T, Li Q, Zhang X, Long R, Wu Y, Wu J, et al. Tox expression decreases with progression of colorectal cancers and is associated with cd4 T-cell density and Fusobacterium nucleatum infection. Hum Pathol. 2018;79: 93-101.

20 Sears CL, Geis AL, Housseau F. Bacteroides fragilis subverts mucosal biology: from symbiont to colon carcinogenesis. J Clin Invest. 2014;124(10):4166-72.

21 Cougnoux A, Dalmasso G, Martinez R, Buc E, Delmas J, Gibold L, et al. Bacterial Genotoxin colibactin promotes colon tumour growth by inducing a senescence-associated secretory phenotype. Gut. 2014;63(12):1932-42.

22 Arthur JC, Perez-Chanona E, Mühlbauer M, Tomkovich S, Uronis JM, Fan TJ, et al. Intestinal inflammation targets cancer-inducing activity of the microbiota. Science. 2012; 338(6103):120-3. 
23 Kato I, Boleij A, Kortman GA, Roelofs R, Djuric Z, Severson RK, et al. Partial associations of dietary iron, smoking and intestinal bacteria with colorectal cancer risk. Nutr Cancer. 2013;65(2):169-77.

24 Tang WH, Hazen SL. The contributory role of gut microbiota in cardiovascular disease. J Clin Invest. 2014;124(10):4204-11.

25 Holmes E, Li JV, Athanasiou T, Ashrafian H, Nicholson JK. Understanding the role of gut microbiome-host metabolic signal disruption in health and disease. Trends Microbiol. 2011; 19(7):349-59.

26 Nakatsu G, Li X, Zhou H, Sheng J, Wong SH, $\mathrm{Wu} \mathrm{WK}$, et al. Gut mucosal microbiome across stages of colorectal carcinogenesis. Nat Commun. 2015;6:8727.

27 Brennan CA, Garrett WS. Fusobacterium nucleatum: symbiont, opportunist and oncobacterium. Nat Rev Microbiol. 2019;17(3):15666.

28 Han YW. Fusobacterium nucleatum: a commensal-turned pathogen. Curr Opin Microbiol. 2015;23:141-7.

29 Raskov H, Burcharth J, Pommergaard HC. Linking gut microbiota to colorectal cancer. J Cancer. 2017;8(17):3378-95.

30 Rubinstein MR, Wang X, Liu W, Hao Y, Cai G, Han YW. Fusobacterium nucleatum promotes colorectal carcinogenesis by modulating $\mathrm{E}$-cadherin/ $\beta$-catenin signaling via its FadA adhesin. Cell Host Microbe. 2013;14(2): 195-206.

31 Gur C, Ibrahim Y, Isaacson B, Yamin R, Abed J, Gamliel M, et al. Binding of the fap2 protein of Fusobacterium nucleatum to human inhibitory receptor tigit protects tumors from immune cell attack. Immunity. 2015;42(2):34455.

32 Grivennikov S, Karin E, Terzic J, Mucida D, Yu GY, Vallabhapurapu S, et al. Il-6 and stat3 are required for survival of intestinal epithelial cells and development of colitis-associated cancer. Cancer Cell. 2009;15(2):103-13.

33 Boleij A, Hechenbleikner EM, Goodwin AC, Badani R, Stein EM, Lazarev MG, et al. The Bacteroides fragilis toxin gene is prevalent in the colon mucosa of colorectal cancer patients. Clin Infect Dis. 2015;60(2):208-15.

34 Thiele Orberg E, Fan H, Tam AJ, Dejea CM, Destefano Shields CE, Wu S, et al. The myeloid immune signature of enterotoxigenic Bacteroides fragilis-induced murine colon tumorigenesis. Mucosal Immunol. 2017;10(2): $421-33$.

35 Wieczorska K, Stolarek M, Stec R. The role of the gut microbiome in colorectal cancer: where are we? Where are we going? Clin Colorectal Cancer. 2019;19(1):5-12.

36 Swidsinski A, Khilkin M, Kerjaschki D, Schreiber S, Ortner M, Weber J, et al. Association between intraepithelial Escherichia coli and colorectal cancer. Gastroenterology. 1998; 115(2):281-6.

37 Sears CL, Garrett WS. Microbes, microbiota, and colon cancer. Cell Host Microbe. 2014; 15(3):317-28.
38 Drewes JL, Housseau F, Sears CL. Sporadic colorectal cancer: microbial contributors to disease prevention, development and therapy. Br J Cancer. 2016;115(3):273-80.

39 Herzer PJ, Inouye S, Inouye M, Whittam TS. Phylogenetic distribution of branched RNAlinked multicopy single-stranded DNA among natural isolates of Escherichia coli. J Bacteriol. 1990;172(11):6175-81.

40 Secher T, Samba-Louaka A, Oswald E, Nougayrède JP. Escherichia coli producing colibactin triggers premature and transmissible senescence in mammalian cells. PloS One. 2013;8(10):e77157.

41 Veziant J, Gagnière J, Jouberton E, Bonnin V, Sauvanet P, Pezet D, et al. Association of colorectal cancer with pathogenic Escherichia coli: focus on mechanisms using optical imaging. World J Clin Oncol. 2016;7(3):293-301.

42 Cuevas-Ramos G, Petit CR, Marcq I, Boury M, Oswald E, Nougayrède JP. Escherichia coli induces DNA damage in vivo and triggers genomic instability in mammalian cells. Proc Natl Acad Sci U S A. 2010;107(25):11537-42.

43 Bonnet M, Buc E, Sauvanet P, Darcha C, Dubois D, Pereira B, et al. Colonization of the human gut by $\mathrm{E}$. coli and colorectal cancer risk. Clin Cancer Res. 2014;20(4):859-67.

44 Pradhan D, Devi Negi V. Stress-induced adaptations in Salmonella: a ground for shaping its pathogenesis. Microbiol Res. 2019;229: 126311.

45 Crum-Cianflone NF. Salmonellosis and the gastrointestinal tract: more than just peanut butter. Curr Gastroenterol Rep. 2008;10(4): 424-31.

46 Keithlin J, Sargeant JM, Thomas MK, Fazil A. Systematic review and meta-analysis of the proportion of non-typhoidal Salmonella cases that develop chronic sequelae. Epidemiol Infect. 2015;143(7):1333-51.

47 Nagaraja V, Eslick GD. Systematic review with meta-analysis: the relationship between chronic Salmonella typhi carrier status and gall-bladder cancer. Aliment Pharmacol Ther. 2014;39(8):745-50.

48 Collier-Hyams LS, Zeng H, Sun J, Tomlinson $\mathrm{AD}, \mathrm{Bao} \mathrm{ZQ}$, Chen $\mathrm{H}$, et al. Cutting edge: Salmonella AvrA effector inhibits the key proinflammatory, anti-apoptotic NF- $\mathrm{kB}$ pathway. J Immunol. 2002;169(6):2846-50.

49 Liu X, Lu R, Wu S, Sun J. Salmonella regulation of intestinal stem cells through the Wnt/ $\beta$-catenin pathway. FEBS Lett. 2010;584(5): 911-6.

50 Hagiya H, Kimura K, Nishi I, Yamamoto N, Yoshida H, Akeda Y, et al. Desulfovibrio desulfuricans bacteremia: a case report and literature review. Anaerobe. 2018;49:112-5.

51 He Q, Hou Q, Wang Y, Li J, Li W, Kwok LY, et al. Comparative genomic analysis of Enterococcus faecalis: insights into their environmental adaptations. BMC Genom. 2018; 19(1):527.
52 Huycke MM, Gaskins HR. Commensal bacteria, redox stress, and colorectal cancer: mechanisms and models. Exp Biol Med. 2004; 229(7):586-97.

53 Khan MS, Ishaq M, Hinson M, Potugari B, Rehman AU. Parvimonas micra bacteremia in a patient with colonic carcinoma. Caspian J Intern Med. 2019;10(4):472-5.

54 Yu J, Feng Q, Wong SH, Zhang D, Liang QY, Qin Y, et al. Metagenomic analysis of faecal microbiome as a tool towards targeted noninvasive biomarkers for colorectal cancer. Gut. 2017;66(1):70-8.

55 Vital M, Howe AC, Tiedje JM. Revealing the bacterial butyrate synthesis pathways by analyzing (meta)genomic data. mBio. 2014;5(2): e00889.

56 Makki K, Deehan EC, Walter J, Bäckhed F. The impact of dietary fiber on gut microbiota in host health and disease. Cell Host Microbe. 2018;23(6):705-15.

57 Singh N, Gurav A, Sivaprakasam S, Brady E, Padia R, Shi H, et al. Activation of Gpr109a receptor for niacin and the commensal metabolite butyrate, suppresses colonic inflammation and carcinogenesis. Immunity. 2014; 40(1):128-39.

58 Yuille S, Reichardt N, Panda S, Dunbar H, Mulder IE. Human gut bacteria as potent class I histone deacetylase inhibitors in vitro through production of butyric acid and valeric acid. PloS One. 2018;13(7):e0201073.

59 Louis P, Hold GL, Flint HJ. The gut microbiota, bacterial metabolites and colorectal cancer. Nat Rev Microbiol. 2014;12(10):661-72.

60 Bernstein C, Holubec H, Bhattacharyya AK, Nguyen H, Payne CM, Zaitlin B, et al. Carcinogenicity of deoxycholate, a secondary bile acid. Arch Toxicol. 2011;85(8):863-71.

61 Elinav E, Nowarski R, Thaiss CA, Hu B, Jin C Flavell RA. Inflammation-induced cancer: crosstalk between tumours, immune cells and microorganisms. Nat Rev Canc. 2013;13(11): 759-71.

62 Flint HJ, Scott KP, Louis P, Duncan SH. The role of the gut microbiota in nutrition and health. Nat Rev Gastroenterol Hepatol. 2012; 9(10):577-89.

63 Yachida S, Mizutani S, Shiroma H, Shiba S, Nakajima T, Sakamoto T, et al. Metagenomic and metabolomic analyses reveal distinct stage-specific phenotypes of the gut microbiota in colorectal cancer. Nat Med. 2019;25(6): 968-76.

64 Bernstein H, Bernstein C, Payne CM, Dvorak K. Bile acids as endogenous etiologic agents in gastrointestinal cancer. World J Gastroenterol. 2009;15(27):3329-40.

65 Suzuki K, Bruce WR. Increase by deoxycholic acid of the colonic nuclear damage induced by known carcinogens in C57BL/6J mice. J Natl Cancer Inst. 1986;76(6):1129-32.

66 Koropatkin NM, Cameron EA, Martens EC. How glycan metabolism shapes the human gut microbiota. Nat Rev Microbiol. 2012; 10(5):323-35. 
67 Gao R, Gao Z, Huang L, Qin H. Gut microbiota and colorectal cancer. Eur J Clin Microbiol Infect Dis. 2017;36(5):757-69.

68 Baxter NT, Zackular JP, Chen GY, Schloss PD. Structure of the gut microbiome following colonization with human feces determines colonic tumor burden. Microbiome. 2014;2:20.

69 Dai Z, Coker OO, Nakatsu G, Wu WKK, Zhao L, Chen Z, et al. Multi-cohort analysis of colorectal cancer metagenome identified altered bacteria across populations and universal bacterial markers. Microbiome. 2018;6(1): 70.

70 Ahn J, Sinha R, Pei Z, Dominianni C, Wu J, Shi J, et al. Human gut microbiome and risk for colorectal cancer. J Natl Cancer Inst. 2013; 105(24):1907-11.

71 Flemer B, Lynch DB, Brown JM, Jeffery IB, Ryan FJ, Claesson MJ, et al. Tumour-associated and non-tumour-associated microbiota in colorectal cancer. Gut. 2017;66(4):633-43.

72 Wang T, Cai G, Qiu Y, Fei N, Zhang M, Pang $\mathrm{X}$, et al. Structural segregation of gut microbiota between colorectal cancer patients and healthy volunteers. ISME J. 2012;6(2):320-9.

73 Allali I, Delgado S, Marron PI, Astudillo A Yeh JJ, Ghazal H, et al. Gut microbiome compositional and functional differences between tumor and non-tumor adjacent tissues from cohorts from the us and spain. Gut Microb. 2015;6(3):161-72.

74 Grahn N, Hmani-Aifa M, Fransén K, Söderkvist P, Monstein HJ. Molecular identification of Helicobacter DNA present in human colorectal adenocarcinomas by $16 \mathrm{~S}$ rDNA PCR amplification and pyrosequencing analysis. J Med Microbiol. 2005;54(Pt 11) 1031-5.

75 Kamada N, Seo SU, Chen GY, Núñez G. Role of the gut microbiota in immunity and inflammatory disease. Nat Rev Immunol. 2013; 13(5):321-35.

76 Chen W, Liu F, Ling Z, Tong X, Xiang C. Human intestinal lumen and mucosa-associated microbiota in patients with colorectal cancer. PloS One. 2012;7(6):e39743.

77 Drewes JL, White JR, Dejea CM, Fathi P, Iyadorai T, Vadivelu J, et al. High-resolution bacterial 16s rRNA gene profile meta-analysis and biofilm status reveal common colorectal cancer consortia. NPJ Biofilms Microbiomes. 2017;3:34

78 Flemer B, Warren RD, Barrett MP, Cisek K, Das A, Jeffery IB, et al. The oral microbiota in colorectal cancer is distinctive and predictive. Gut. 2018;67:1454-63.
79 Routy B, Le Chatelier E, Derosa L, Duong CPM, Alou MT, Daillère R, et al. Gut microbiome influences efficacy of pd-1-based immunotherapy against epithelial tumors. Science. 2018;359:91-7.

80 Mira-Pascual L, Cabrera-Rubio R, Ocon S, Costales P, Parra A, Suarez A, et al. Microbial mucosal colonic shifts associated with the development of colorectal cancer reveal the presence of different bacterial and archaeal biomarkers. J Gastroenterol. 2015;50(2):16779.

81 Kostic AD, Chun E, Robertson L, Glickman JN, Gallini CA, Michaud M, et al. Fusobacterium nucleatum potentiates intestinal tumorigenesis and modulates the tumor-immune microenvironment. Cell Host Microbe. 2013; 14(2):207-15

82 Nougayrède JP, Homburg S, Taieb F, Boury M, Brzuszkiewicz E, Gottschalk G, et al. Escherichia coli induces DNA double-strand breaks in eukaryotic cells. Science. 2006; 313(5788):848-51.

83 Huycke MM, Moore DR. In vivo production of hydroxyl radical by Enterococcus faecalis colonizing the intestinal tract using aromatic hydroxylation. Free Radic Biol Med. 2002; 33(6):818-26.

84 Cooke MS, Evans MD, Dizdaroglu M, Lunec J. Oxidative DNA damage: mechanisms, mutation, and disease. FASEB J. 2003;17(10): 1195-214.

85 Taieb F, Petit C, Nougayrède JP, Oswald E. The enterobacterial genotoxins: cytolethal distending toxin and colibactin. EcoSal Plus. 2016;7(1).

86 Papapietro O, Teatero S, Thanabalasuriar A, Yuki K, Diez E, Zhu L, et al. R-spondin 2 signalling mediates susceptibility to fatal infectious diarrhoea. Nat Commun. 2013;4:18981916.

87 Rhee KJ, Wu S, Wu X, Huso DL, Karim B, Franco AA, et al. Induction of persistent colitis by a human commensal, enterotoxigenic Bacteroides fragilis, in wild-type C57BL/6 mice. Infect Immun. 2009;77(4):1708-18.

88 Plaza-Diaz J, Gomez-Llorente C, Fontana L, Gil A. Modulation of immunity and inflammatory gene expression in the gut, in inflammatory diseases of the gut and in the liver by probiotics. World J Gastroenterol. 2014; 20(42):15632-49.

89 Grenham S, Clarke G, Cryan JF, Dinan TG Brain-gut-microbe communication in health and disease. Front Physiol. 2011;2:94.

$90 \mathrm{Wu}$ S, Rhee KJ, Albesiano E, Rabizadeh S, Wu $\mathrm{X}$, Yen HR, et al. A human colonic commensal promotes colon tumorigenesis via activation of T helper type $17 \mathrm{~T}$ cell responses. Nat Med. 2009;15(9):1016-22.
91 Housseau F, Wu S, Wick EC, Fan H, Wu X, Llosa NJ, et al. Redundant innate and adaptive sources of IL17 production drive colon tumorigenesis. Cancer Res. 2016;76(8): 2115-24.

92 Grivennikov SI, Wang K, Mucida D, Stewart CA, Schnabl B, Jauch D, et al. Adenomalinked barrier defects and microbial products drive IL-23/IL-17-mediated tumour growth. Nature. 2012;491(7423):254-8.

93 Foltánková V, Legartová S, Kozubek S, Hofer M, Bártová E. DNA-damage response in chromatin of ribosomal genes and the surrounding genome. Gene. 2013;522(2): 156-67.

94 Sarhadi V, Lahti L, Saberi F, Youssef O, Kokkola A, Karla T, et al. Gut microbiota and host gene mutations in colorectal cancer patients and controls of Iranian and Finnish origin. Anticancer Res. 2020;40(3):1325-34.

95 Kopper L, Mihalik R. [Cell death and tumor formation]. Orv Hetil. 1999;140(23):12839.

96 Kasai C, Sugimoto K, Moritani I, Tanaka J, Oya Y, Inoue H, et al. Comparison of human gut microbiota in control subjects and patients with colorectal carcinoma in adenoma: terminal restriction fragment length polymorphism and next-generation sequencing analyses. Oncol Rep. 2016;35(1): 325-33.

97 Zackular JP, Rogers MA, Ruffin MT, Schloss $\mathrm{PD}$. The human gut microbiome as a screening tool for colorectal cancer. Canc Prev Res. 2014;7:1112-21.

98 Lee DW, Han SW, Kang JK, Bae JM, Kim $\mathrm{HP}$, Won JK, et al. Association between Fusobacterium nucleatum, pathway mutation, and patient prognosis in colorectal cancer. Ann Surg Oncol. 2018;25(11):3389-95.

99 Komiya Y, Shimomura Y, Higurashi T, Sugi Y, Arimoto J, Umezawa S, et al. Patients with colorectal cancer have identical strains of Fusobacterium nucleatum in their colorectal cancer and oral cavity. Gut. 2019;68(7): $1335-7$.

100 Colov EP, Degett TH, Raskov H, Gögenur I The impact of the gut microbiota on prognosis after surgery for colorectal cancer: a systematic review and meta-analysis. APMIS. 2020;128(2):162-76.

101 Shah MS, DeSantis TZ, Weinmaier T, McMurdie PJ, Cope JL, Altrichter A, et al. Leveraging sequence-based faecal microbial community survey data to identify a composite biomarker for colorectal cancer. Gut. 2018;67:882-91. 